\title{
BMJ open Qualitative analysis of patients' feedback from a PROMs survey of cancer patients in England
}

\author{
Jessica Corner, ${ }^{1}$ Richard Wagland, ${ }^{1}$ Adam Glaser, ${ }^{2}$ Sir Mike Richards ${ }^{3}$
}

To cite: Corner J, Wagland $\mathrm{R}$, Glaser A, et al. Qualitative analysis of patients' feedback from a PROMs survey of cancer patients in England. BMJ Open 2013;3:e002316. doi:10.1136/bmjopen-2012002316

\section{- Prepublication history for this paper are available online. To view these files please visit the journal online (http://dx.doi.org/10.1136/ bmjopen-2012-002316).}

Received 6 November 2012 Revised 27 February 2013 Accepted 4 March 2013

This final article is available for use under the terms of the Creative Commons Attribution Non-Commercial 2.0 Licence; see http://bmjopen.bmj.com

\section{${ }^{1}$ Faculty of Health Sciences, University of Southampton, Southampton, UK ${ }^{2}$ Department of Paediatric Oncology, Leeds General Infirmary, Leeds, UK ${ }^{3}$ Department of Health, Cancer Policy Team, London, UK}

Correspondence to Professor Jessica Corner; J.L.Corner@soton.ac.uk

\section{SLinked}

- http://dx.doi.org/10.1136/ bmjopen-2012-002317

\section{ABSTRACT}

Objective: This study examined how free-text comments from cancer survivors could complement formal patient-reported outcome measures (PROMs), as part of the England PROMs survey programme for cancer.

Design: A qualitative content analysis was conducted of responses to a single open-ended free-text question placed at the end of the cross-sectional populationbased postal questionnaire.

Setting: Individuals were identified through three UK Cancer Registries and questionnaires were posted to their home addresses.

Participants: A random sample of individuals $(n=4992)$ diagnosed with breast, colorectal, non-Hodgkins lymphoma or prostate cancer at 1, 2, 3 and 5 years earlier.

Results: 3300 participants completed the survey $(68 \%$ response rate). Of these $1056(32 \%)$ completed the free-text comments box, indicating a high level of commitment to provide written feedback on patient experience. Almost a fifth (19\%) related experiences of excellent care during the treatment phase, with only $8 \%$ reporting negative experiences. This contrasted with experiences of care after primary cancer treatment where the majority were negative. Factors impacting negatively upon patient-reported outcomes included the emotional impact of cancer; poor experiences of treatment and care; comorbidities, treatment side effects, social difficulties and inadequate preparation for a wide range of sometimes long-lasting on-going physical and psychological problems. Mediating factors assisting recovery incorporated both professional-led factors, such as quality of preparation for anticipated problems and aftercare services, and participant-led factors, such as learning from other cancer survivors and self-learning through trial and error. The support of friends and family was also a factor in participants' outcomes.

Conclusions: This analysis of free-text comments complements quantitative analysis of PROMs measure's by illuminating relationships between factors that impact on quality of life (QoL) and indicate why cancer patients may experience significantly worse QoL than the general population. The data suggest more systematic preparation and aftercare for individuals to self-manage post-treatment problems might improve QoL outcomes among cancer survivors.

\section{ARTICLE SUMMARY}

Article focus

- Examination of the potential for free-text comments to complement formal patient-reported outcomes.

- Investigation into the potential for the collection of long-term data relating to health outcomes of cancer survivors and experiences of care.

- Address the limited attention that perceived quality of care or on-going needs have received in previous large-scale surveys.

Key messages

- Collecting free-text comments on patient experiences is a valuable addition to the use of patientreported outcome measures (PROMs) survey measures.

- Some individuals reported that they were poorly prepared for life after cancer treatment.

- Data reinforced the need for greater emphasis by cancer services to support individuals to selfmanage following completion of cancer treatment.

Strengths and limitations of this study

- Free-text comments elaborated upon experiences indicated within the PROMs, suggesting they are a valid representation of the views of participants.

- Strong themes emerged from the data, with clearly inter-related associations, enabling development of a framework encompassing all responses that illuminates the findings of the quantitative survey.

- Broad phrasing of the question elicited a varied range of participant responses which may reduce representativeness, validity and interpretation, and recall and response bias may be present.

\section{INTRODUCTION}

Life expectancy for individuals diagnosed with cancer has doubled over the last 40 years and the majority of individuals with common cancers can expect to live for 10 years or more. ${ }^{1}$ These developments are transforming the experience of cancer from 
one where in the past, the disease signalled a potentially life-threatening illness, to one where today we are beginning to think of cancer as a long-term or chronic condition. ${ }^{2}$ As a result it is increasingly important that the problems patients face following treatment, and how these can be addressed are understood. There is growing recognition that people living with cancer need support during the survival phase of their illness, ${ }^{3-7}$ and, as previous qualitative research has shown, many cancer survivors experience a broad range of significant on-going physical, psychological and social needs. ${ }^{8-11}$ Other, now seminal, studies show how individuals undergo a process of recovery, adjustment and renegotiation of identity as cancer is assimilated as a lifechanging and long-term illness, and individuals respond to the stigma of cancer, a process that is not addressed by conventional 'medical' management approaches. ${ }^{12}$ More recently a multidimensional model of recovery following cancer treatment has been proposed. ${ }^{6}$

Increasing recognition that patient-reported outcome measures (PROMs) can convey important information for assessing the overall cancer burden and the effectiveness of interventions has led to much work to develop psychometrically sound and clinically meaningful PROMs applicable to a range of chronic conditions, ${ }^{13} 14$ although more qualitative work has been called for to examine the processes that contribute to outcomes. ${ }^{13}$ Much of this work has been carried out by the Patient-Reported Outcomes Measurement Information System cooperative group. ${ }^{15}{ }^{16}$ At the same time, a growing number of surveys across the USA and Canada are being undertaken to collect data on cancer survivors, although as a recently published systematic review confirms,${ }^{17}$ perceived quality of care or on-going needs have received little attention in such surveys. There has also to date been little research to systematically determine quality-of-life (QoL)-related outcomes on a health system-wide basis over the long term or as to how health systems can best respond to needs identified for patients following primary treatment for cancer. ${ }^{8} 1718$ Within the UK the measurement of outcomes, improving patient experience and enhancing QoL for people with a longterm condition is a department of health priority. ${ }^{19}$

This paper reports qualitative findings from the first PROMs Survey of Cancer Survivors, to complement the quantitative findings from the survey's formal PROMs measures. ${ }^{20}$ The study was established as part of the National Cancer Survivorship Initiative (NCSI) to evaluate patients' experiences of cancer care in England. The aim was to establish that reliable information, in the form of PROMs, could be systematically collected from cancer survivors on both treatment and care on a health system-wide basis and over the long term, with a view to service improvement initiatives aimed at better addressing patients' needs. Patients responding to the survey were invited to comment on their experiences to provide context to quantitative responses, and give a deeper understanding of the nature of the problems they face, framed from their own perspectives and priorities. The responses stand alongside the highly structured PROMs measures that are framed from the perspective of researchers, and provide rich insights into patients' needs which can be used to support improvement initiatives. $^{21}$

\section{OBJECTIVES}

The study explored how participants utilised the opportunity to make free-text comments as part of the England PROMs survey programme for cancer, and examined how emerging themes complement formal PROMs to provide understanding of QoL issues for cancer survivors.

\section{METHODS}

\section{Study design}

A cross-sectional population-based postal survey of a random sample of individuals identified as having been diagnosed with breast, colorectal, non-Hodgkins lymphoma or prostate cancer $1,2,3$ and 5 years earlier. The sample was chosen to include both common and rarer cancers and a spectrum of cancer survivors over the period of 5 years following cancer diagnosis and initial cancer treatment.

\section{Cohort identification}

As cancer survivors may no longer be under the care of cancer treatment centres the study population was identified through Cancer Registries. Cancer registries (Thames, Eastern and West Midlands) provided information on all recorded cases of the four included cancer diagnoses registered as being diagnosed 1, 2, 3 and 5 years ago.

Cases were excluded if they were, under the age of 16 years, deceased, non-National Health Service (NHS) patients, not known to have a UK address. The total of 4992 patients was equally distributed between the cancer groups and cohort years, giving an initial sample of 312 in each cohort year and cancer group. Patients were randomly selected and are therefore representative of all cases.

\section{Questionnaire design and content}

Questionnaires were developed for each cancer group. Content included the EQ5D, ${ }^{22}{ }^{23}$ the Social Difficulties Inventory, ${ }^{24}{ }^{25}$ Functional Assessment of Cancer Therapy $^{26}$ and a free-text comments box. Cognitive testing was performed and alterations were made to the questionnaires for postcognitive testing.

\section{Survey process}

Patients consented to taking part in the survey by returning questionnaires and declined by not returning them, or by returning a blank questionnaire. Two reminders were sent to non-responders. 


\section{Ethics and governance}

An approval was given by the National Information Governance Board. Ethical review was not sought for this survey as it was performed as a service evaluation. ${ }^{27}$ Survey participants had access to a telephone support line that could be used to discuss any issues raised by the survey.

\section{Analysis}

A single open-ended free-text question was placed at the end of the questionnaire, following the closed questions, with the header: "If you have anything else you would like to tell us about living with and beyond cancer, please do so here."

Findings from the analysis of quantitative data from the PROMs measures are reported by Glaser $e t$ al. ${ }^{28}$ The analysis of the responses to the free-text section of the questionnaire aimed to examine how these may shed light on why outcomes reported may have arisen and whether any of the issues raised by participants warrant a response from the healthcare system to address issues or problems raised. The qualitative analysis involved developing a thematic framework that captured all the qualitative data, alongside participants' tumour groups and time from diagnosis, to identify associations between separate themes and from which defensible inferences could be made. ${ }^{29} 30$ The concepts of validity and trustworthiness within qualitative research are understood in terms of the credibility of the analytical process. ${ }^{29}{ }^{30}$ Comments were analysed using the NVivo software package, ${ }^{31}$ using a two-stage approach. Such analyses of comments within quantitative surveys has been previously used to research health service issues. ${ }^{32}$

The first stage of the analysis sought to discover the different ways that participants chose to answer the freetext question and develop a representative thematic framework. After careful reading and data immersion, ${ }^{33}$ one researcher (RW) conducted a conventional qualitative content analysis, ${ }^{34}$ coding all comments and identifying broad themes within an initial framework. Categories were developed inductively, were exhaustive and mutually exclusive. The preliminary coding schema was discussed with a second researcher (JC) and revised before all data within each code was re-examined.

A second level of analysis was then undertaken to identify patterns and relationships in the data, that is, where many participants suggested there were relationships between factors that negatively impacted on their QoL and indicated potentially mediating factors. The researchers began to develop a tentative conceptual model of the experiences of participants that might help qualify, clarify and support the findings of the quantitative survey. ${ }^{29}$ Throughout its development the model underwent a thorough process of repeated testing against the data, to examine and challenge its capacity to represent all participant experiences. Several versions of the model were proposed, tested, discussed within the team and subsequently revised, to ensure the validity of the final model presented in figure $1 .{ }^{30}$

The number of participants writing about a particular theme are not seen as 'representative' of the views of the sample as a whole as they reflect what individuals chose to write. Nevertheless, simple counts are used to illustrate the proportion of comments that addressed particular themes, and when an issue was raised frequently, weight was attributed to this as reflecting an important element of experience. A limited number of direct quotes from participants have been used to convey some important themes.

\section{FINDINGS}

Questionnaires were sent to 4992 patients, 126 (2.5\%) of these had moved or died prior to receiving the questionnaire so the final sample size was 4866 . In total, 3300 completed questionnaires were received with an overall response rate of $68 \%$, of whom a third $(1056,32 \%)$ added material to the free-text comments box. Table 1 shows that participants completing the free-text box included very similar numbers of participants from each of the cancer sites (though with slightly more breast cancer survivors), male and female participants (537 and 519, respectively) and age groups.

As might be expected from a broadly phrased open question, the way in which participants chose to respond was diverse and varied in length and depth of information provided. As table 2 shows, within the first phase of the analytic process, the thematic framework categorised participant's responses into three over-riding themes: experiences of cancer diagnosis and treatment; experiences of living beyond cancer and issues unrelated to the experience of living beyond cancer. For the purposes of this paper, only findings within the first two themes will be presented, with most emphasis given to the second. Patterns in the views and experiences among participants were observed in the data and, during the second phase of the analytical process, a tentative model was developed (figure 1). Six factors appeared to impact negatively on patient-reported outcomes, while mediating factors that assisted recovery incorporated both professional-led and patient-led factors. Although developed from the data within this study this model has parallels with the conceptual model of self-management and self-management support following primary cancer treatment. ${ }^{6}$

Many participants $(n=209)$ related experiences of excellent or very good care during the diagnostic and treatment phases of their disease. Positive comments included appreciation of a timely diagnosis through screening programmes or general practitioners (GPs) acting promptly to investigate reported symptoms, excellent or very good general care from the NHS, and coordinated care between staff and hospital departments. Some of the most positive comments reflected a sense of gratitude to particularly efficient and caring healthcare 


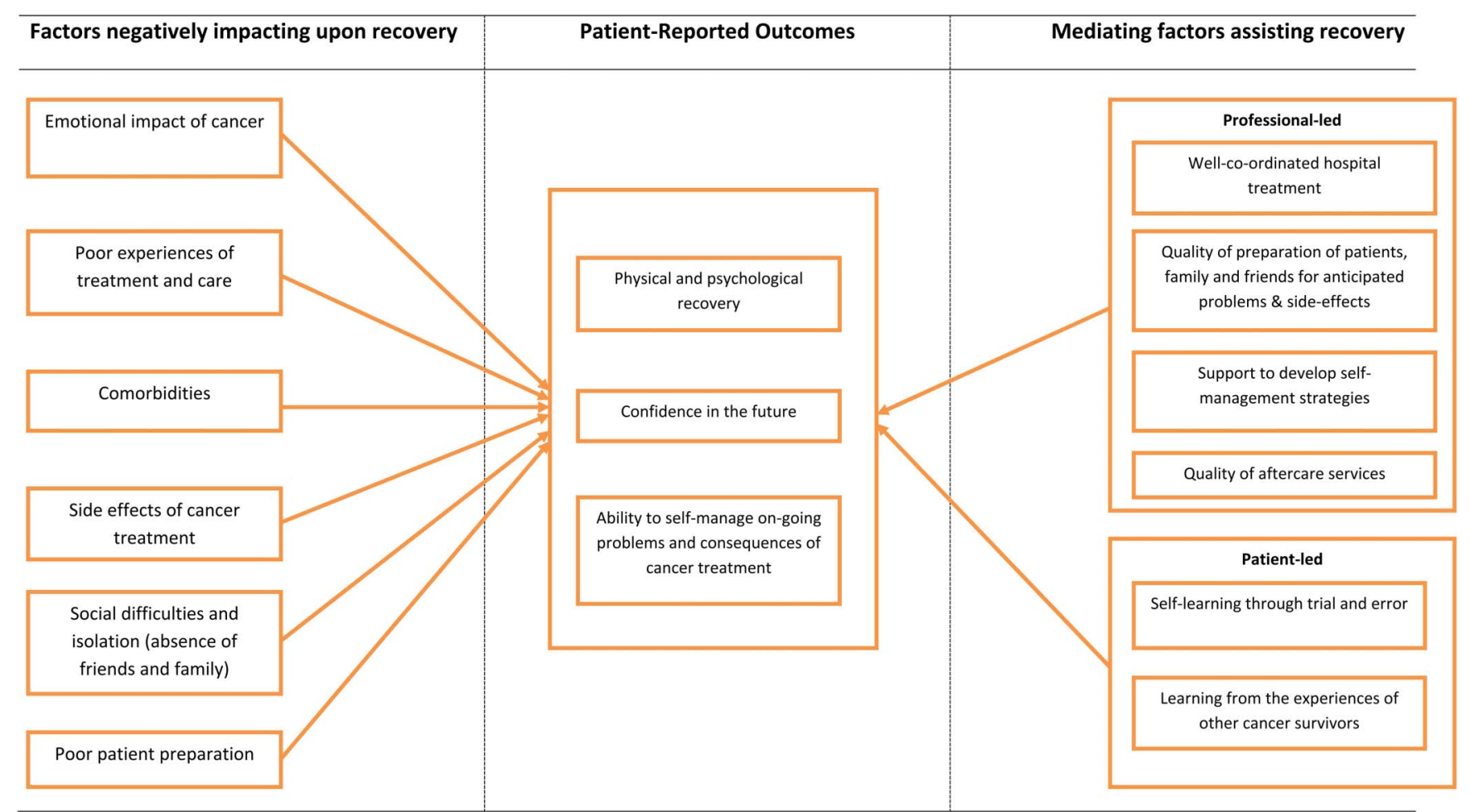

Figure 1 A tentative model to indicate factors impacting on quality of life outcomes.

professionals. The trust such experiences engendered contributed positively to mental and physical recovery. Of those participants reflecting negative experiences $(n=81)$ the most commonly reported involved: 'unacceptable' diagnostic and treatment delays, including delayed GP referrals to specialist consultations; instances of poor inpatient care; poorly coordinated treatment often perceived to be caused by unreliable hospital administrative processes; repeated appointment cancellations and delays; and lack of emotional support from some staff. All such experiences potentially contributed to the stress of cancer treatment.

A large number of participants $(n=172)$ described how various comorbidities they suffered impacted on their experience of living beyond cancer, how they added to their physical, psychological and social difficulties following completion of cancer treatment and affected their QoL, and how comorbidities had often been exacerbated by their cancer treatment. Reported comorbidities varied widely but hypertension, arthritis,

Table 1 Age and gender of survey participants providing free-text comments by Cancer Group

\begin{tabular}{|c|c|c|c|c|c|c|c|c|c|c|}
\hline \multirow[b]{2}{*}{ Characteristic } & \multicolumn{2}{|c|}{ Breast $(n=290)$} & \multicolumn{2}{|c|}{$\begin{array}{l}\text { Colorectal } \\
(n=258)\end{array}$} & \multicolumn{2}{|c|}{$\begin{array}{l}\text { Non-Hodgkin's } \\
\text { lymphoma } \\
(n=253)\end{array}$} & \multicolumn{2}{|c|}{$\begin{array}{l}\text { Prostate } \\
(n=255)\end{array}$} & \multicolumn{2}{|c|}{ Total $(n=1056)$} \\
\hline & $\mathrm{n}$ & Per cent & $\mathrm{n}$ & Per cent & $\mathrm{n}$ & Per cent & $\mathrm{n}$ & Per cent & $\mathrm{n}$ & Per cent \\
\hline \multicolumn{11}{|l|}{ Sex } \\
\hline Male & 1 & $<0.1$ & 143 & 13.5 & 138 & 13.1 & 255 & 24.1 & 537 & 50.9 \\
\hline Female & 289 & 27.3 & 115 & 10.8 & 115 & 10.8 & 0 & 0 & 519 & 49.1 \\
\hline \multicolumn{11}{|l|}{ Age } \\
\hline Under 50 & 42 & 3.9 & 11 & 1.0 & 34 & 3.2 & 1 & $<0.1$ & 88 & 8.3 \\
\hline $50-64$ & 120 & 11.3 & 59 & 5.6 & 81 & 7.7 & 44 & 4.2 & 304 & 28.8 \\
\hline $65-74$ & 77 & 7.3 & 83 & 7.8 & 69 & 6.5 & 108 & 10.2 & 337 & 31.9 \\
\hline $75+$ & 51 & 4.8 & 105 & 9.9 & 69 & 6.5 & 102 & 9.7 & 327 & 30.9 \\
\hline \multicolumn{11}{|c|}{ Time from diagnosis (years) } \\
\hline 1 & 71 & 24.4 & 57 & 21.9 & 68 & 26.8 & 69 & 27.0 & 265 & 25.1 \\
\hline 2 & 70 & 24.1 & 68 & 26.3 & 67 & 26.4 & 67 & 26.2 & 272 & 25.8 \\
\hline 3 & 67 & 23.1 & 69 & 26.7 & 63 & 24.9 & 50 & 19.6 & 249 & 23.6 \\
\hline 5 & 82 & 28.3 & 64 & 24.6 & 55 & 21.7 & 69 & 27.0 & 270 & 25.5 \\
\hline
\end{tabular}

Survey participants who provided comments to the text box $(n=1056)$ represented $32 \%$ of all survey participants $(n=4992)$. 
Table 2 Thematic framework of the experience of living with and beyond cancer derived from free-text responses to the PROMs survey questionnaire

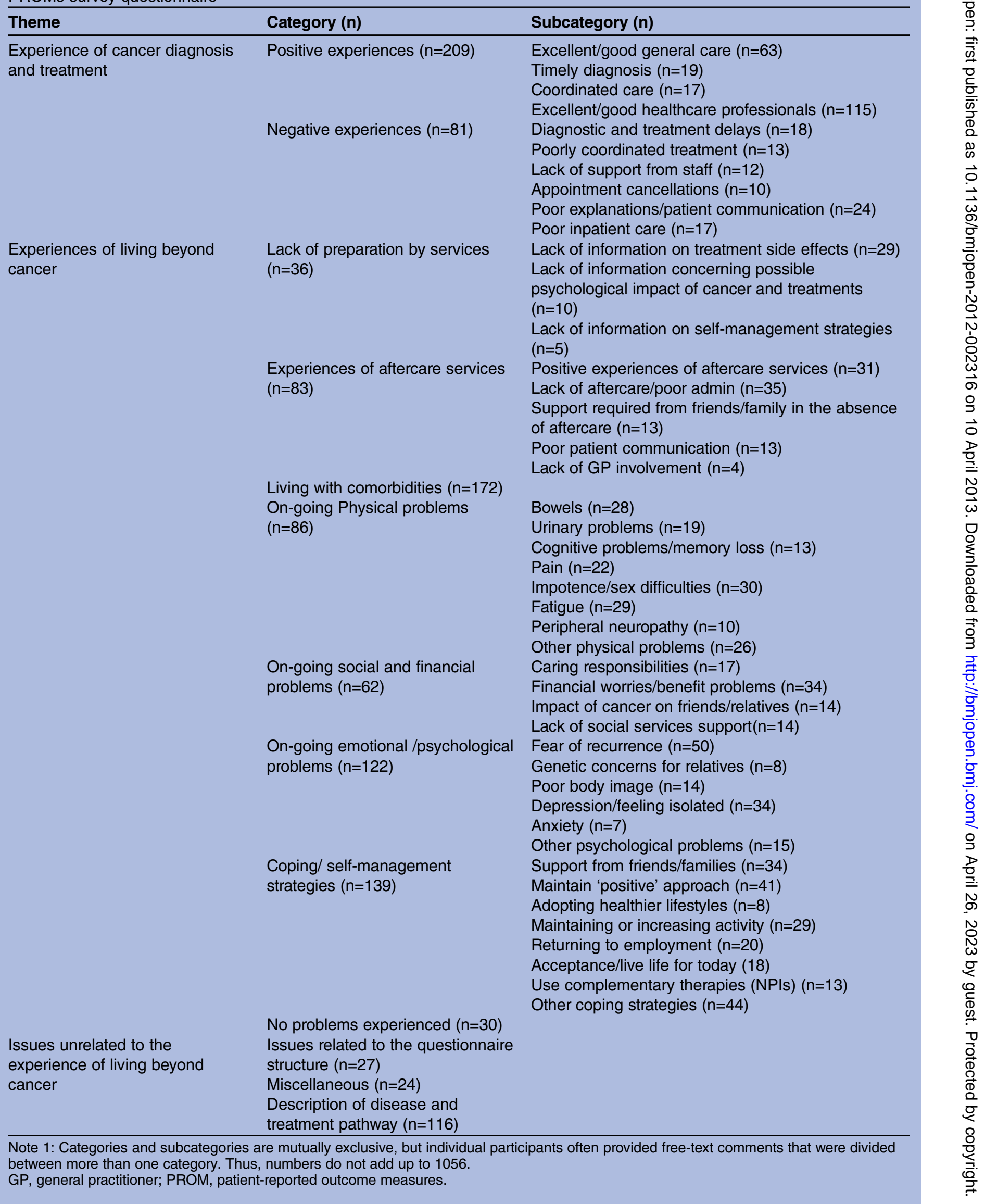


osteoporosis, long-term back pain and general problems relating to old age were predominant.

A wide range of on-going physical, psychological and social problems were reported by participants that were side effects of cancer treatment and which impacted greatly on QoL and mirror responses to the quantitative PROMs. Physical problems were described by 86 participants, and, as found in the quantitative data, those most commonly reported included problems with bowel and urinary incontinence; constipation; cognitive problems and diminished memory; pain; impotence and sexual difficulties; fatigue and peripheral neuropathy. Participants related how these frequently had a considerable impact restricting activities such as employment, travel and social activities, and were reported across all four tumour groups and timepoints from cancer diagnosis. Social factors were identified $(n=62)$ that either caused or exacerbated the stresses of coping with a cancer diagnosis, including caring responsibilities, financial worries and the need to return to work. Concerns about the impact of the diagnosis on family and friends were common; especially worries about who would look after dependents should the cancer return. Similarly, financial worries were present for those not able to work, especially among self-employed participants, and contributed to feelings of depression and anxiety about the future.

Various on-going psychological problems were described by 122 participants, as a consequence of having had cancer (eg, fear of recurrence, difficulty planning ahead, fears that the disease will be hereditary and affect their children); and as a consequence of the on-going physical problems experienced (eg, poor body image following breast surgery or stoma formation, depression caused by sexual difficulties following surgery for bowel or hormone therapy for prostate cancer). Problems caused by cancer treatment were often either permanent or took far longer to diminish than participants had envisaged or had been advised, and there were similar numbers of participants describing such on-going problems 5 years from their diagnosis as participants diagnosed more recently. Again, this reflects scores from the quantitative PROMs measures, and many participants expressed surprise and exasperation at the duration of these problems:

\footnotetext{
Thirteen months after surgery, I had a reversal (stoma). I was not given any advice or information as to what to expect regarding my bowel movements. To this day, I have to have a very limited diet, and choice of food if I do not want to spend all day on the toilet. I was never told that my bowel movements would not return to normal. I do not travel very far from home!
}

An important theme in the data was that some participants $(n=36)$ perceived they had received a lack of preparation concerning the potential impact of cancer and its treatment. Inadequate preparation was found to have several dimensions: not being informed about the possible physical side effects of cancer treatments (that lymphoedema may follow breast surgery, that chemotherapy could lead to peripheral neuropathy, cognitive problems and bring on early menopause); being unaware of the potential psychological impact of both experiencing a cancer diagnosis and of treatment side effects, and when these might occur; not being provided with equipment to manage practical difficulties and not being advised of potential coping strategies to adopt. Participants indicated that such lack of preparation could greatly contribute to the negative impact that on-going physical and psychological problems had on QoL, but inadequate preparation also represented an on-going unmet need that may create an unnecessary additional cost for the NHS.

I am concerned about the lack of information available in oncology units regarding long term after effects of treatments with chemotherapy. Tens of thousands of patients have been given chemo over many years and a significant proportion including myself must have sought advice on symptoms occurring sometime after treatment has stopped. It would appear that there has been no recorded effort to collate this wealth of information. This must have caused unnecessary stress to patients and cost the NHS a considerable amount through additional patient appointments.

The issue of preparation is not restricted to the responsibility of clinical teams: rather it is a system-wide issue and includes the information provided by pharmaceutical companies concerning known side effects of drugs.

Since the very first dose of [drug name] I have been afflicted with bruising and sometimes bleeding on my arms from the slightest bump with door handles and supermarket shelves. I also suffer from tiredness and joint pain of various degrees of severity...the leaflet that accompanies [drug name] carries the date November 1997. After years of the life of a newish drug could not the manufacturers be prevailed on to bring up to date information that comes in every pack that cramp is a side effect.

There was also dissatisfaction concerning the adequacy of products designed to help manage the physical side effects of treatment and the personal expense involved.

Incontinence was initially devastating but reduced over 4 months and by 6 months I was fully continent at night and by day needed only a small pad...my GP arranged provision of [Product name] male pads which were excellent. Due to administrative change the supplier was changed to [different product] incontinence pads which are useless as supplied because the penis post-radical prostatectomy shortens and cannot fit into the pad pocket...My GP nurse tells me they can only supply [product name] pads. This is not satisfactory...there is a need for improvement in NHS support. 
Participants reported that a lack of information about possible treatment side effects sometimes caused unnecessary concern, even if these are not serious, because they were unexpected. Moreover, being insufficiently prepared also reportedly impacted on the ability of some participants to make informed decisions about treatment options. For example, one breast cancer survivor reported that she had received insufficient advice regarding fertility changes and the options available, and that the information booklet she received was reportedly outdated. Other participants stated that had they been provided with full information about the treatments they were going to receive they might not have proceeded with them.

I had colorectal surgery, it's side effects affected my life badly. I should have been warned/advised about those side effects to decide. If I knew about them I would not go ahead with the surgery.

Many participants $(n=83)$ related experiences of aftercare, the majority reporting negative experiences. Some of these participants contrasted the good hospital treatment they had received with a lack of aftercare, which left them feeling isolated by the healthcare system once primary treatment was completed. Participants $(n=34)$ often described the support they had received from family and friends after treatment providing practical help with housework and child care, emotional and financial support. However, in the absence of aftercare some participants $(n=13)$ related ways in which their families and friends had helped with nursing care and administering medication.

With regards to social services, more help etc. They did nothing for me. We/I never saw a nurse or home help. Even my daughter had to administer my injections after having [instruction] from the cancer nurses.

We received no support...My partner had to change the drain bags for me as I was unable to do this myself.

The lack of aftercare often coincided with the point in recovery when the psychological impact of cancer and treatment was greatest. However, the tone of many comments is one of the enduring problems over many years without any form of support, with a few instances in which the free-text box was used specifically to ask for help. A need for greater emotional support for both physical and psychological problems as part of aftercare is articulated; especially as such problems often fail to diminish over time. Participants have a need to talk, ask questions and regain self-confidence.

Should be more aftercare even when you are in remission. Once you have all your treatment you are left on your own to come to terms with what has happened. You don't always want a medical team, just someone you can talk with.
Several participants noted that without informal support from friends and family individuals would have greater need of support from the NHS and allied agencies.

Support of my wife and family made it easier to deal with. I feel those facing this alone or who are afraid to talk about it or even say the word cancer would need more help and support.

Participants who described significant emotional impact of having had cancer, or reported on-going treatment side effects with which they were struggling to cope, often also described experiencing a lack of patient preparation and aftercare. Conversely, by examining the comments of participants who report good experience and who have learnt to manage on-going problems it is possible to identify the various factors that helped mitigate what might potentially have led to poor life quality. The mitigating factors fell into two groups: professional-led and survivor-led.

Survivors describing good experiences often praised the well-coordinated care they had received while undergoing investigations and treatment and had been sufficiently prepared by health professionals for problems associated with cancer and treatment side effects. Professional-led mitigating factors also included quality aftercare, provided by named healthcare professionals especially clinical nurse specialists, with whom survivors and their families could remain in contact and discuss problems as they arose, and who supported the development of self-management strategies. This level of continuity often addressed the hiatus that many experienced at the end of their treatment, helped reduce fear of recurrence and increased confidence in the future.

Many survivors who coped well with on-going problems had reportedly discovered self-management strategies for themselves through a process of trial and error, often with the support of friends or family members, or through talking to others with similar experiences, rather than having had them explained by professionals. Strategies adopted by participants included: focusing upon the 'positive' aspects of life $(n=41)$; adopting healthier lifestyles $(\mathrm{n}=8)$; maintaining or gradually increasing activity $(\mathrm{n}=29)$ as a way of getting life back to normal; returning to employment or voluntary work $(n=20)$; and the use of psychosocial and complementary therapies $(n=13)$. The teaching of potential strategies to manage physical problems such as bowel and urinary incontinence, fatigue and sexual difficulties transcends both patient preparation and aftercare, and several participants argued that affordable access to interventions such as counselling and talking therapies should be available to address issues like altered body image and fear of recurrence. 


\section{DISCUSSION}

This analysis of free-text comments within the pilot PROMs survey complements quantitative analysis of the formal measures by illuminating relationships between factors that impact on QoL or mitigate against negative effects. These insights can then be passed on to NHS providers to help improve the quality of patient experience. This approach adopted alongside formal PROMs measures, demonstrates that individuals actively engage with the opportunity to provide comments relating to their experiences, providing data relating to 'why?' health outcomes are reported in formal measures, and illuminating insights from statistical analysis. ${ }^{21}$ While quantitative analysis of the survey's closed questions indicated that some participants appeared to report poor QoL relative to the general population, and that a substantial proportion of individuals report on-going health needs, ${ }^{28} 35$ these data do not shed light on the experiences of treatment care, aftercare following cancer treatment or on what might improve health outcomes or patient experiences. The fact that one in three of those that completed the PROMs questionnaires took the opportunity to provide such feedback provides a significant resource and, while this cannot be viewed as necessarily 'representative' of patients experiences' more generally, they nevertheless provide rich insights into these, and are data not available in other large-scale surveys of cancer survivors. ${ }^{17}$

The free-text comments thus complement the quantitative results by allowing participants to indicate important issues of priority to them. Through both the formal $\mathrm{PROMs}^{20} 28$ and the free-text comments many participants described the impact of treatment upon comorbidities, and greater incidence of poor health has previously been shown to exist among cancer survivors with comorbid conditions. ${ }^{3563637}$ Many on-going problems and concerns were also identified, and, while these are similar to those reported in other studies, there is little current evidence concerning the prevalence and nature of many such problems. ${ }^{6}{ }^{17} 18$ Within this study similar numbers of participants provided comments on such issues across all four tumour groups and timepoints from cancer diagnosis, indicating these are widespread and enduring. ${ }^{3}$ By identifying the patterns of factors that might negatively impact on QoL within the English cancer population, on a basis greater than the single trial or observational study, this study assists policy-makers to explore ways to target system improvement efforts.

An important theme among participants was a perceived lack of preparation about what to expect with regard to potential physical and psychological impact of the cancer and treatment side effects, which is supported by previous research showing that cancer survivors do not receive optimal levels of information about life beyond cancer treatment. ${ }^{38} 39$ Another theme was a dissatisfaction with aftercare services among some participants, who often felt 'cut adrift' by the health system after the 'intense' period of treatment and were left feeling vulnerable. ${ }^{3} 40$ Participants reported they lacked information regarding possible strategies for dealing with their on-going problems, while research evidence to date regarding the effectiveness of many such potential self-management approaches remains limited. ${ }^{6} 1718$ Recent evidence suggests that patients want more information concerning long-term effects of cancer and treatment, and to be provided with psychosocial support and strategies for self-management. ${ }^{41}$ There is a clear need for consideration to be given as to how to improve care for these patients. ${ }^{42} \mathrm{~A}$ fundamental shift is currently underway in the way that cancer survivors are supported after treatment, as part of the Department of Health for England NCSI, ${ }^{7}{ }^{10}$ and other similar initiatives worldwide. ${ }^{17}$ These data indicate that the DH PROMs programme for cancer has the potential for providing data to evaluate the effectiveness of such initiatives in relation to conventional health measurement tools in the form of PROMs and also through feedback framed by patients themselves. ${ }^{43}$

\section{CONCLUSION}

Comments provided by individuals participating in the England Department of Health, Cancer PROMs survey reinforce the need for greater emphasis to be given by cancer services to supporting individuals to manage following completion of cancer treatment. This is in keeping with the priorities of the Department of Health NCSI, ${ }^{10}$ and similar initiatives in other countries. ${ }^{44}$ Preparation and support for life after cancer treatment should be consistently provided for all cancer survivors. Some individuals are currently ill-prepared for the physical consequences of some treatments for cancer and the psychological aftermath of receiving a diagnosis of a potentially lifethreatening illness. The absence of early intervention to investigate and treat serious on-going physical problems that result from certain treatments for cancer is unacceptable. The potential for early intervention and more systematic preparation for individuals to self-manage posttreatment problems should be explored.

Contributors JC and MR conceived the idea of the study and acquisition of the data. JC, RW, MR and AG were responsible for the design of the study. $\mathrm{RW}$ and $\mathrm{JC}$ were responsible for undertaking data analysis, with input from $M R$ and $A G$. The initial draft of the paper was produced by JC and RW, and circulated repeatedly between all authors for critical revision. All authors were involved with interpretation of the results, read and approved the final manuscript.

Funding Grateful acknowledgement is made to Macmillan Cancer Support for providing funding for open access publication.

Competing interests Adam Glaser and Mike Richards were employed within the Cancer Policy Team within the Department of Health, England while the reported study was performed.

Provenance and peer review Not commissioned; externally peer reviewed.

Data sharing statement Original data are available on request from the Department of Health. Please contact the corresponding author in the first instance. 


\section{REFERENCES}

1. Cancer Research UK. Cancer survival rates-trends. 2007. http:// info.cancerresearchuk.org/cancerstats/survival/fiveyear/ (accessed 20 Sept 2012).

2. Tritter JQ, Calnan M. Cancer as a chronic illness? Reconsidering categorization and exploring experience. Eur J Cancer Care 2002;11:161-5

3. Armes J, Crowe M, Colbourne L, et al. Patients' supportive care needs beyond the end of cancer treatment: a prospective, longitudinal survey. J Clini Oncol 2009;27:6172-9.

4. Corner J. Addressing the needs of cancer survivors: issues and challenges. Expert Rev Pharmacoecon Outcomes Res 2008;8:443-51.

5. Elliott J, Fallows A, Staetsky L, et al. The health and well-being of cancer survivors in the UK: findings from a population-based survey. Br J Cancer 2011;105:S11-20.

6. Foster C, Fenlon D. Recovery and self-management support following primary cancer treatment. Br J Cancer 2011;105:S21-8.

7. Davies NJ, Batehup L. Towards a personalised approach to aftercare: a review of cancer follow-up in the UK. $J$ Cancer Surviv 2011;5:142-51.

8. Foster $\mathrm{C}$, Wright $\mathrm{D}$, Hill $\mathrm{H}$, et al. Psychosocial implications of living 5 years or more following a cancer diagnosis: a systematic review of the research evidence. Eur J Cancer Care 2009;18:223-47.

9. Richards M, Corner J, Maher J. The National Cancer Survivorship Initiative: new and emerging evidence on the on-going needs of cancer survivors. B J Cancer 2011;105:S1-4.

10. Department of Health, Macmillan Cancer Support, NHS Improvement. The National Cancer Survivorship Initiative Vision. Department of Health, 2010. http://www.dh.gov.uk/en/ Publicationsandstatistics/Publications/PublicationsPolicy AndGuidance/DH 111230 (accessed 20 Sept 2012).

11. Rivers BM, August EM, Gwede CK, et al. Psychosocial issues related to sexual functioning among African-American prostate cancer survivors and their spouses. Psychooncology 2011;20:106-10.

12. Mathieson $\mathrm{CM}$, Stam HJ. Renegotiating identity: cancer narratives. Sociol Health IIIn 1995;12:283-306.

13. Lipscomb J, Gotay CC, Snyder CF. Patient-reported outcomes in cancer: a review of recent research and policy initiatives. CA Cancer J Clin 2007;57:278-300.

14. Lipscomb J, Reeve BB, Clauser SB, et al. Patient-reported outcomes assessment in cancer trials: taking stock, moving forward. $J$ Clin Oncol 2007;25:5133-40.

15. Cella D, Yount S, Rothrock N, et al. The patient-reported outcomes measurement information system (PROMIS): progress of an $\mathrm{NIH}$ Roadmap cooperative group during its first two years. Med Care 2007;45(5 Suppl 1):S3-11.

16. Garcia S, Cella D, Clauser S, et al. Standardizing patient-reported outcomes assessment in cancer clinical trials: a patient-reported outcomes measurement information system initiative. J Clin Oncol 2007;25:5106-12.

17. Lerro CC, Stein KD, Smith $\mathrm{T}$, et al. A systematic review of large-scale surveys of cancer survivors conducted in North America 2000-2011. J Cancer Surviv 2012;6:115-45.

18. Richardson A, Addington-Hall J, Amir Z, et al. Knowledge, ignorance and priorities for research in key areas of cancer survivorship: findings from a scoping review. Br J Cancer 2011;105:S82-94.

19. Department of Health. The NHS Outcomes Framework 2012/13; December 2011. Https://www.wp.dh.gov.uk/publications/files/2012 11/121109-NHS-Outcomes-Framework-2013-14.pdf (accessed 20 Sept 2013).

20. Department of Health-Quality Health. Quality of life of cancer survivors in England: report on a pilot survey using Patient Reported Outcome Measures (PROMS). London, 2012. http://www.dh.gov.uk/ health/2012/12/cancer-proms/ (accessed 20 Sept 2012).

21. Robert G, Cormwell J, Brearley S, et al. What matters most to patients? Developing the evidence base of measuring and improving patient experience. NHS Institute for Innovation and Improvement, November 2011. Http://www.institute.nhs.uk/patient_expereince/ guide/what_matters_to_patients\%3F.pdf (accessed July 2012).
22. Group TE. EuroQol-a new facility for the measurement of healthrelated quality of life. Health Policy 1990;16:199-208.

23. Herdman M, Gudex C, Lloyd A, et al. Development and preliminary testing of the new five-level version of EQ-5D (EQ-5D-5L). Qual Life Res 2011;20:1727-36.

24. Wright EP, Kiely M, Johnston C, et al. Development and evaluation of an instrument to assess social difficulties in routine oncology practice. Qual Life Res 2005;14:373-86.

25. Wright $P$, Smith $A B$, Keding $A$, et al. The Social Difficulties Inventory (SDI): development of subscales and scoring guidance for staff. Psycho-Oncology 2011;20:36-43.

26. Webster K, Cella D, Yost K. The Functional Assessment of Chronic IIIness Therapy (FACIT) Measurement System:properties, applications and interpretation. Health and Quality of Life Outcomes 2003;1:79

27. National Patient Safety Agency. Defining research-guidance from the National Research Ethics Service, London, NRES. 2010. http:// www.nres.nhs.uk/applications/guidance/research-guidance/? entryid62=66985 (accessed 20 Sept 2012).

28. Glaser AW, Fraser LK, Corner J, et al. Patient Reported Outcomes of Cancer Survivors in England. BMJ Open 2013;3:e002317. doi:10.1136/bmjopen-2012-002317.

29. Bryman A, Burgess RG. Analyzing qualitative data. London: Routledge, 1994.

30. Seale C. The quality of qualitative research. London: Sage, 1999

31. Gibbs GR. Qualitative data analysis: explorations with NVivo. Buckingham: Open University, 2002.

32. Richards SH, Campbell JL, Walshaw E, et al. A multi-method analysis of free-text comments from the UK General Medical Council Colleague Questionnaires. Med Educ 2009;43:757-66.

33. Miles MB, Huberman AM. Quantitative data analysis: an expanded sourcebook. Thousand Oaks, CA: Sage, 1994.

34. Hsieh H-F, Shannon SE. Three approaches to qualitative content analysis. Qual Health Res 2005;15:1277-88.

35. Department of Health-Quality Health. Quality of life of cancer survivors: Report on a pilot survey using patient reported outcome measures (PROMS). http://www.dh.gov.uk/health/2012/12/ cancer-proms/ (accessed 8 Feb 2013).

36. Hewitt M, Greenfield S, Stovall E, eds. From cancer patient to cancer survivor: Lost in transition. Washington, DC: National Academies Press, 2006.

37. Jefford M, Karahalios E, Pollard A, et al. Survivorship issues following treatment completion-results from focus groups with Australian cancer survivors and health professionals. J Cancer Surviv 2008;2:20-32.

38. Ayanian JZ, Zaslavsky AM, Arora NK, et al. Patients' experiences with care for lung cancer and colorectal cancer: findings from the cancer care outcomes research and surveillance consortium. J Clini Oncol 2010;28:4154-61.

39. Harrison SE, Watson EK, Ward AM, et al. Cancer survivors experiences of discharge from hospital follow-up. Eur J Cancer Care 2012;21:390-7.

40. Pennry E, Mallett J. A preliminary study of patient's perceptions of follow-up after treatment for breast cancer. Eur J Oncol Nurs 2000;4:138-45.

41. Rutten LJF, Arora NK, Bakos AD, et al. Information needs and sources of information among cancer patients: a systematic review of research (1980-2003). Patient Educ Couns 2005;57:250-61.

42. Rowland J, Hewitt M, Ganz P. Cancer survivorship: a new challenge in delivering quality cancer care. J Clin Oncol 2006;24:5101-4.

43. Corner J, Wagland R. National cancer survivorship initiative: text analysis of patients' free text comments: final report. Southampton: University of Southampton; 2012. http://www.dh.gov.uk/health/2013/ 01/responses-cancer-survivors (accessed 25 Jan 2013).

44. Fitch MI. Looking to the Future: Cancer Survivorship in Canada. Canadian Partnership Against Cancer. June 2011. www.caot.ca/ pdfs/PIF/P2\%20PIF.pdf

45. Hewitt M, Rowland JH, Yancik R. Cancer survivors in the United States: age, health and disability. J Geronto 2003;58:82-91. 